\title{
Mechanisms and Dynamics of Mineral Dissolution: a New Kinetic Monte Carlo Model
}

\author{
Pablo Martin, Hegoi Manzano* and Jorge S. Dolado*
}

Mr. P. Martin

Building Technologies Division Tecnalia, Parque Tecnológico de Bizkaia, Astondo Bidea, Edificio 700, Derio, Bizkaia, Spain

Dr. H. Manzano

Department of Condensed Matter Physics, University of the Basque Country UPV/EHU, Barrio Sarriena s/n, 48940, Leioa, Bizkaia, Spain

E-mail: Hegoi.manzano@ehu.eus

Dr. J. S. Dolado

Materials Physics Center - Centro de Física de Materiales CSIC-UPV/EHU, Paseo Manuel de Lardizabal, 5, 20018 San Sebastian, Spain

Donostia International Physics Center, Paseo Manuel Lardizabal 3, 20018, San Sebastián, Spain

Faculty of Civil Engineering and Geosciences, Delft University of Technology, Stevinweg 1, 2628 CN, Delft, The Netherlands

E-mail: Jorge_dolado002@ehu.eus

Abstract: Mineral dissolution is a fundamental process in Earth and materials science. It is controlled by the complex interplay of atomic level mechanisms like adatoms and terraces removal, pit opening and spontaneous vacancy creation that can be gradually activated at different energies. Though the development of a comprehensive atomistic model is key to go deeper into the understanding of this phenomenon, existing models have failed to reproduce the abrupt dependence of the dissolution rate with the Gibbs free energy $(\Delta G)$. Herein we present a new atomistic Kinetic Monte Carlo (KMC) model that invoking the microscopic reversibility of chemical reactions captures the experimentally observed sigmoid dependence of the dissolution rate and provides new insights on the concomitant dissolution mechanisms. As a salient result, the model predicts the possible existence of unreported close-to-equilibrium dissolution modes where spontaneous vacancies creation and pit opening can occur before adatom and terrace removal. 


\section{Introduction}

Mineral dissolution has high relevance in countless important geophysical phenomena like soil formation ${ }^{[1,2]}$, water and petrol reservoir stability ${ }^{[3,4]}$ or carbon sequestration ${ }^{[5-8]}$, among others. To describe these phenomena is crucial to completely understand dissolution process, yet it presents some unanswered issues. The dissolution of a solid material is a complex process which is simultaneously affected by the solid surface topology (exposed surface, nature of the atomic bonds, presence of impurities, defects, etc) and the nature of the surrounding solution. Indeed, the driving force of dissolution is controlled by the Gibbs free energy $(\Delta G)$, which is closely related to the ion activity in the solvent $^{[9-16]}$. From experiments a sigmoidal shape dependence between dissolution rate and $\Delta G$ is generally obtained (see Figure 1c) ${ }^{[10,12-15,17,18]}$. Different regions in the sigmoidal function seem to be governed by different dissolution mechanisms depending on the mineral cohesive energy and $\Delta G$. These mechanisms can be observed experimentally when vertical scanning interferometry and atomic force or scanning electron microscopy are used to follow the surface topography during dissolution ${ }^{[9,10,15,19,20]}$. In a typical curve like the one portrayed in Figure 1c, three mechanisms with different dissolution rates can be distinguished:

- Mechanism I. At low negative $\Delta G$ adatoms and terraces dissolve leading to a flat surface. The dissolution rate is very low due to the close position to equilibrium state.

- Mechanism II. As $\Delta G$ decreases, vacancies and dislocations of the system become dissolution cores leading to pit opening and step retreat.

- Mechanism III. As $\Delta G$ becomes even lower, any surface atom can become a dissolution core. However, this only occurs in minerals with low cohesive energy.

The three dissolution mechanisms are supposed to be additive and must coexist, that is, it is not possible to have mechanism II without mechanism I, or mechanism III without II and I, and they take place simultaneously. The value of $\Delta G$ where the onset of dissolution rate takes place due to mechanism II is called critical $\Delta G\left(\Delta G_{\text {crit }}\right)$.

Computational methods have played an important role in the last thirty years to give basic atomic level explanation to dissolution. In 1986 Lasaga and Blum studied the etch pits formation due to dislocations using a Monte Carlo (MC) method ${ }^{[21]}$. This implied the beginning of the so called flickering-bond model development where the dissolution rate is defined by the elementary bond-forming bond-breaking reactions ${ }^{[19,22]}$. About ten years later ${ }^{[23]}$, the generalized use of Kinetic Monte Carlo (KMC) method involved a great progress due to its faster computing speed. For example, Liang et al. used KMC to consider explicitly the effects of structure and surface topography at the molecular scale 
in calcite ${ }^{[24]}$, and more recently, Kurganskaya and Luttge have used KMC to study silicate $^{[25]}$ and carbonate ${ }^{[26]}$ minerals dissolution with atomistic detail at far from equilibrium conditions.

Common KMC models based on Transition State Theory (TST) consider that one atom, molecule or coarse grained is removed from the system ${ }^{[11]}$. The rate of this dissolution event, $r_{\mathrm{D}}$, depends on its dissolution reaction energy barrier $E_{\mathrm{D}}$.

$$
r_{\mathrm{D}}=\frac{k_{\mathrm{B}} \cdot T}{\hbar} \cdot \exp \left(-\frac{E_{\mathrm{D}} \cdot N_{\mathrm{n}}}{k_{\mathrm{B}} \cdot T}\right)
$$

where $k_{B}$ is the Boltzmann constant, $\hbar$ the Planck constant, $T$ the temperature and $N_{n}$ the number of first neighbours. $k_{\mathrm{B}} \cdot T / \hbar$ is the commonly named fundamental frequency $f_{\mathrm{f}}$. Despite their simplicity, TST-based KMC models allow to study minerals dissolution at far from equilibrium conditions. In fact, they capture the inherent topographies associated to the dissolution mechanisms ${ }^{[25-28]}$, they permit the analysis of effects like dislocations ${ }^{[27,28]}$, grain sizes ${ }^{[29]}$, particular mineral structures ${ }^{[25,26]}$, etc, and even they reproduce the experimentally observed pulsating frequency at the nanoscale ${ }^{[30]}$.

It is generally accepted from the above mechanisms that the origin of the sigmoidal behaviour with $\Delta G$ arises from the interplay between the mineral nanoscale topography and the $\Delta G$ itself. Previous works have regarded the study of dissolution rate dependence with $\Delta G$ by MC method by considering dissolution and precipitation separately ${ }^{[2,31,32]}$, failing to reproduce the dissolution mechanisms over the whole $\Delta G$ range. In this work an extension to the model has been proposed based on TST and microscopic reversibility (Equations 2 and 3). The usual mineral-water reaction equation proposed by Lasaga ${ }^{[21,22]}$ is complemented, considering dissolution and precipitation events independently. For each event the rate depends on its specific energy barriers of dissolution or precipitation and the vicinity of the dissolved molecule.

For simplicity, the model has been applied to study the dissolution of a Kossel crystal. A Kossel crystal, or Terrace Ledge Kink system (TLK) is a simple mineral structure consisting of a cubic structure with six first neighbours ${ }^{[33]}$ (see Figure 1a). Despite the simplicity of this system, it ensures enough topographical details so as to reproduce the mechanisms attributed to the dissolution process.

The paper is organized as follows: First, the net dissolution rate dependence with the main parameters of the model, i.e. the dissolution and precipitation energy barriers, has been explored. As said before, the model is able to reproduce the sigmoid dissolution rate function with $\Delta G$ and the intrinsic mechanisms. Second, these microscopic parameters have been related to macroscopic parameters, like the dissolution onset $\Delta G_{\text {crit }}$, or the effective activation energy $E_{\mathrm{a}}$, readily accessible by experimental means. Finally, experimental data for several minerals have been fitted, finding a reasonable good agreement in spite of the structural limits of using a simple Kossel crystal. 
a

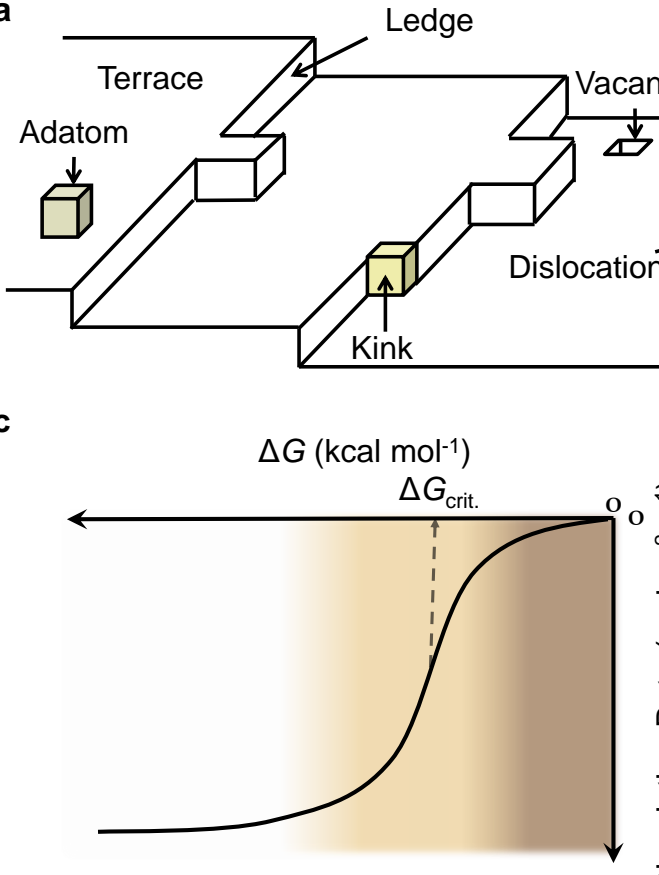

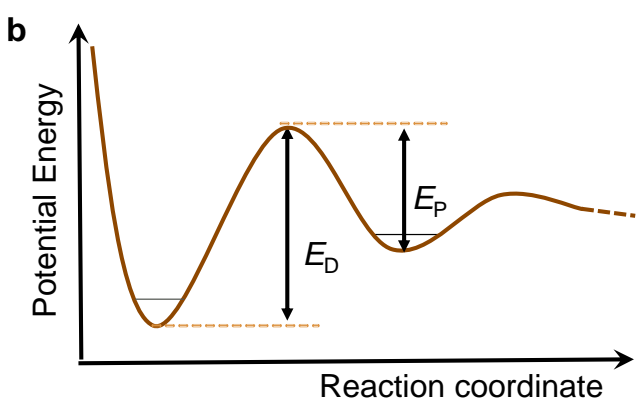

d

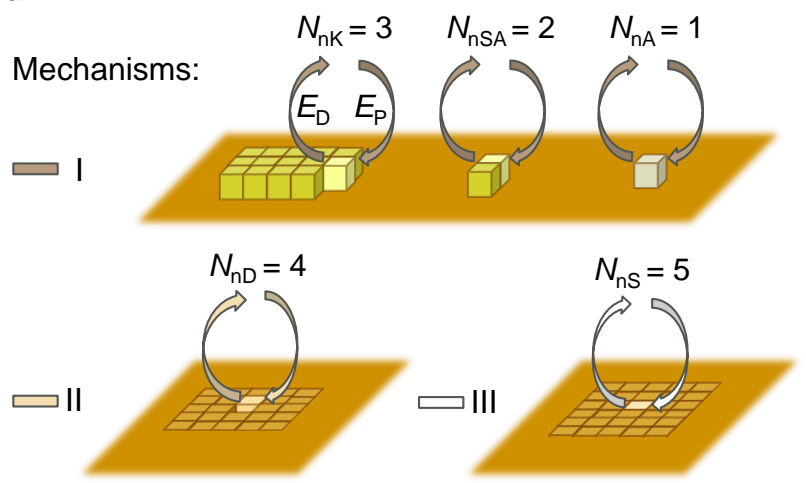

Figure 1: Schematic representation of mineral dissolution processes. a, Representation of a Kossel's surface with different topographic defects. Dotted lines represent an infinite hole. b, Potential energy curve in a typical dissolution process. Solid lines represent the fundamental frequency for the potential wells. c, Dissolution pathways versus $\Delta G$ of a mineral. The onset in dissolution rate due to mechanism II is given at $\Delta G_{\text {crit }}$. $\mathrm{d}$, The three generic dissolution mechanisms ${ }^{[17]}$. $N_{\mathrm{nK}}$ coordination number for a kink atom, $N_{\text {nSA }}$ for a step adatom, $N_{\text {nA }}$ for an adatom, $N_{\mathrm{nD}}$ for an atom close to a dislocation or step atom and $N_{\mathrm{nS}}$ for a surface atom. 


\subsection{Model description}

As is mentioned above, mineral dissolution depends on the equilibrium between the solid and the solution, represented by $\Delta G$. At a microscopic level, the net dissolution rate is the result of the equilibrium between dissolution and precipitation. On the one hand, dissolution is related to mineral cohesive energy. On the other hand, precipitation varies with both mineral nature and saturation grade of solvent. Dissolved atoms tends to stay close to the mineral, which leads to a high supersaturation state close to the surface, or what it is the same, to a low negative Gibbs free energy $(\Delta G)$ between mineral and solvent. Under these conditions, close to the surface there could be the possibility for an already dissolved atom or molecule to reform its bonds and come back to the material ${ }^{[21]}$. In equilibrium, dissolution and precipitation processes have the same probability, what is known as microscopic reversibility ${ }^{[34]}$. Bandstra and Brantley ${ }^{[35]}$ used this idea to develop a simple 2-D Ising model to study dissolution dependence with $\Delta G$, achieving a good description. Following that idea, here it is suggested to divide the net dissolution into two processes described by the following equations:

$$
\left\{\begin{array}{l}
r_{\mathrm{D}}=f_{\mathrm{f}} \cdot \exp \left(-\frac{E_{\mathrm{D}} \cdot N_{\mathrm{n}}}{k_{\mathrm{B}} \cdot T}\right) \\
r_{\mathrm{P}}=f_{\mathrm{f}} \cdot \exp \left(-\frac{E_{\mathrm{P}} \cdot N_{\mathrm{n}}-\Delta G^{*}}{k_{\mathrm{B}} \cdot T}\right)
\end{array}\right.
$$

Where $N_{\mathrm{n}}$ is the number of first neighbours, $E_{\mathrm{D}}$ and $E_{\mathrm{P}}$ are the energy barriers for a dissolution and a precipitation event respectively (see Figure 1), and $\Delta G^{*}$ is the local Gibbs free energy.

With this model both dissolution and precipitation are taken into account, and together they describe the net dissolution process. Equation 2 is the classic KMC equation that has been widely used in dissolution kinetics ${ }^{[25-30]}$. Dissolution is assumed to be intrinsic for a mineral and its topography, via $E_{\mathrm{D}}$ and $N_{\mathrm{n}}$ respectively, and it does not depend on $\Delta G^{*}$. Equation 3 accounts for precipitation phenomenon, and takes into account the dependence with the solute concentration by means of $\Delta G^{*}$, and similarly to the dissolution rate equation, the nature of the mineral and topography is considered via $E_{\mathrm{P}}$ and $N_{\mathrm{n}}$.

Two aspects of the model must be remarked. First, when the system is very far from equilibrium $\left(\Delta G^{*} \rightarrow-\infty\right)$ the precipitation rate becomes negligible and the proposed model is then reduced to the usual Arrhenius Equation 1. Second, if the net dissolution rate $r_{\mathrm{D}}-r_{\mathrm{P}}$ is calculated and $E_{\mathrm{D}}=E_{\mathrm{P}}$ and no $N_{\mathrm{n}}$ dependence is considered, the general expression for the normal TST is recovered (Equation 4).

$$
r_{\mathrm{D}}-r_{\mathrm{P}}=A \cdot\left(1-\exp \left(\frac{\Delta G^{*}}{k_{\mathrm{B}} \cdot T}\right)\right)
$$


where $A$ is a constant.

In Equation 3 the local $\Delta G^{*}$ must be related to the macroscopic $\Delta G$. For that, the kink site is taken as a reference for the mineral dissolution ${ }^{[11,33,34]}$ (see Figure 1a). It is a peculiar position: independently of the mineral composition an atom in this position has always half the number of bonds that in the bulk, and therefore its energy is half. Indeed, it is observed experimentally that the sublimation energy of a crystal corresponds to the change of the internal energy when detaching a kink atom ${ }^{[11,33,34]}$. With this site as reference, $\Delta G^{*}$ and $\Delta G$ for a crystal are related by the following expression if the activity is set to the unity (Equation 5) ${ }^{[33,35] \text { : }}$

$$
\Delta G=\left(\Delta G^{*}-b\right) \cdot N_{\mathrm{nK}}
$$

where $N_{\mathrm{nK}}$ is the coordination of a kink site; in a Kossel crystal $N_{\mathrm{nK}}=3$. The constant $b$ can be determined by considering that equilibrium is reached during dissolution when no kink atoms can dissolve $r_{\mathrm{D}}=r_{\mathrm{P}}$, and hence the macroscopic $\Delta G=0$ (see eqs. 6,7 ):

$$
r_{\mathrm{D}}\left(N_{\mathrm{n}}=N_{\mathrm{nK}}\right)=r_{\mathrm{P}}\left(N_{\mathrm{n}}=N_{\mathrm{nK}}\right) \rightarrow \Delta G=0
$$

Expressing the result in $k_{\mathrm{B}} T$ units, the constant $b$ is:

$$
b=N_{\mathrm{nK}} \cdot\left(E_{\mathrm{P}}-E_{\mathrm{D}}\right)
$$

\section{Methods}

The system under study consist in a Kossel crystal with 240 x 240 x 8 sites for a total of $\sim 460800$ with periodic boundary conditions. With a typical distance between atoms of $2.5 \AA^{[36]}$, the system has a size of $\sim 60 \times 60 \mathrm{~nm}$. Mineral topography and surface are key factors in the dissolution mechanisms. To simulate a mineral as real as possible all the different topographic defects showed in Figure 1a have been included. On the surface, a random number of terraces, vacancies, and adatoms have been represented.

The system has two initial dislocations. This equates, for the system size, to a dislocation density of $\sim 5.5 \cdot 10^{10} \mathrm{~cm}^{-2}$. This value lies into the known dislocation density range

for minerals: $10^{6}-10^{10} \mathrm{~cm}^{-2}[37]$. The disposition of the dislocations varies with the crystallographic plane considered. In this paper the simplest case is going to be considered; the $\{100\},\{010\}$ and $\{001\}$ planes with perpendicular dislocations as it can be appreciated in Figure 1a. An on-lattice KMC home code has been developed using $\mathrm{C}++11$ language. Only surface atoms are supposed to react since they are in contact with the solvent, therefore there are no possible events for a bulk atom.

A major problem in simulations close to equilibrium conditions is that sites undergo dissolution and precipitation reactions rapidly. These fast events happen continuously, inducing an exponential increase on the use of computing resources, blocking the simulation. Therefore a methodology to unstuck it is necessary, skipping repeating and computing 
consuming processes. There are several algorithms to avoid such issue ${ }^{[38-40]}$, yet a new one has been used based on Poisson processes statistics, explained in detail in the supplementary information. Briefly, for an atom attempting to dissolve, it is possible to do an estimation of the probability for that atom to truly leave the mineral taking into account both dissolving and precipitation rates. System times are then recalculated based on that probability and plenty of simulation time is saved depending on $\Delta G$ value and mineral cohesive energy. While far from equilibrium conditions this does not make any difference, close to it, in a highly cohesive mineral, simulations are about $10^{4}$ times faster.

The next step is to set the values of the model parameters. The activation energy $E_{\mathrm{a}}$ for a dissolution process of a typical mineral lies in the range of $30 \lesssim E_{\mathrm{a}} \lesssim 85 \mathrm{~kJ} \mathrm{~mol}^{-1[28]}$. Expressing this in $k_{\mathrm{B}} T$ units $\left(k_{\mathrm{B}} T=2.494 \mathrm{~kJ} \mathrm{~mol}^{-1}\right.$ at $\left.300 \mathrm{~K}\right), 12 \lesssim E_{\mathrm{a}} \lesssim 34$. In the model $E_{\mathrm{D}}$ represents the bond breaking energy barrier, which in a Kossel crystal with an average of three broken bonds during a dissolution event, should correspond to $E_{\mathrm{a}} / 3$. Hence, simulations with $E_{\mathrm{D}}=4.0,7.0,9.0,12.0 k_{\mathrm{B}} T$ units have been done. Several $\Delta G$ values covering the three mechanism zones have been taken starting from $\Delta G=0$ and with finer focus on the $\Delta G_{\text {crit }}$ onset. For the energy barrier of precipitation events a value of $E_{\mathrm{P}}=1.0 k_{\mathrm{B}} T$ units is taken. The fundamental frequency in minerals can range between $\sim 10^{11}$ up to $\sim 10^{14} \mathrm{~s}^{-1[33,41]}$. A value of $f_{\mathrm{f}}=4 \cdot 10^{13} \mathrm{~s}^{-1}$ at $T=300 \mathrm{~K}$ is considered for this work. Herein, the presented results are the average of 5 simulations for each condition unless the contrary is specified.

\section{Results}

\subsection{Dissolution rate as a function of $\Delta G$}

First, the effect of $E_{\mathrm{D}}$ on the dissolution rate as a function of $\Delta G$ is tested. Simulations with $E_{\mathrm{D}}=4.0,7.0,9.0,12.0 k_{\mathrm{B}} T$ units are shown in Figure 2a. It is remarkable that the curves have the sigmoidal shape found experimentally. To the best of our knowledge this is the first model that reproduces the whole $\Delta G$ dependence and captures the inherent mechanism and its topography. Close to $\Delta G=0$, in mechanism I region, very low dissolution takes place since only adatoms and terraces are dissolved. Then dissolution slowly increases until $\Delta G$ is low enough to allow pit opening or mechanism II, leading to a sharp onset. Finally a steady dissolution rate is obtained at very low $\Delta G$ values when mechanism III governs dissolution if $E_{\mathrm{D}}$ is low enough. It is important to notice that the relationship between $\Delta G_{\text {crit }}$ and $E_{\mathrm{D}}$ matches what is seen in experiments; the higher the $E_{\mathrm{D}}$, the lower the $\Delta G_{\text {crit }}$. Another fact of agreement with experiments is the maximum dissolution rate far from equilibrium: the higher the $E_{\mathrm{D}}$, the lower negative rate.

In Figure $2 \mathrm{a}$ the cases $E_{\mathrm{D}}=7.0 k_{\mathrm{B}} T$ and $E_{\mathrm{P}}=7.0 k_{\mathrm{B}} T$ and $E_{\mathrm{P}}=10.0 k_{\mathrm{B}} T$ are studied to show how $E_{\mathrm{P}}$ affects mechanisms and to underline that the TST curve can also be reproduced by the model. Two interesting phenomena should be emphasized. On one hand, mechanisms II and III can take place very close to equilibrium conditions. On the 

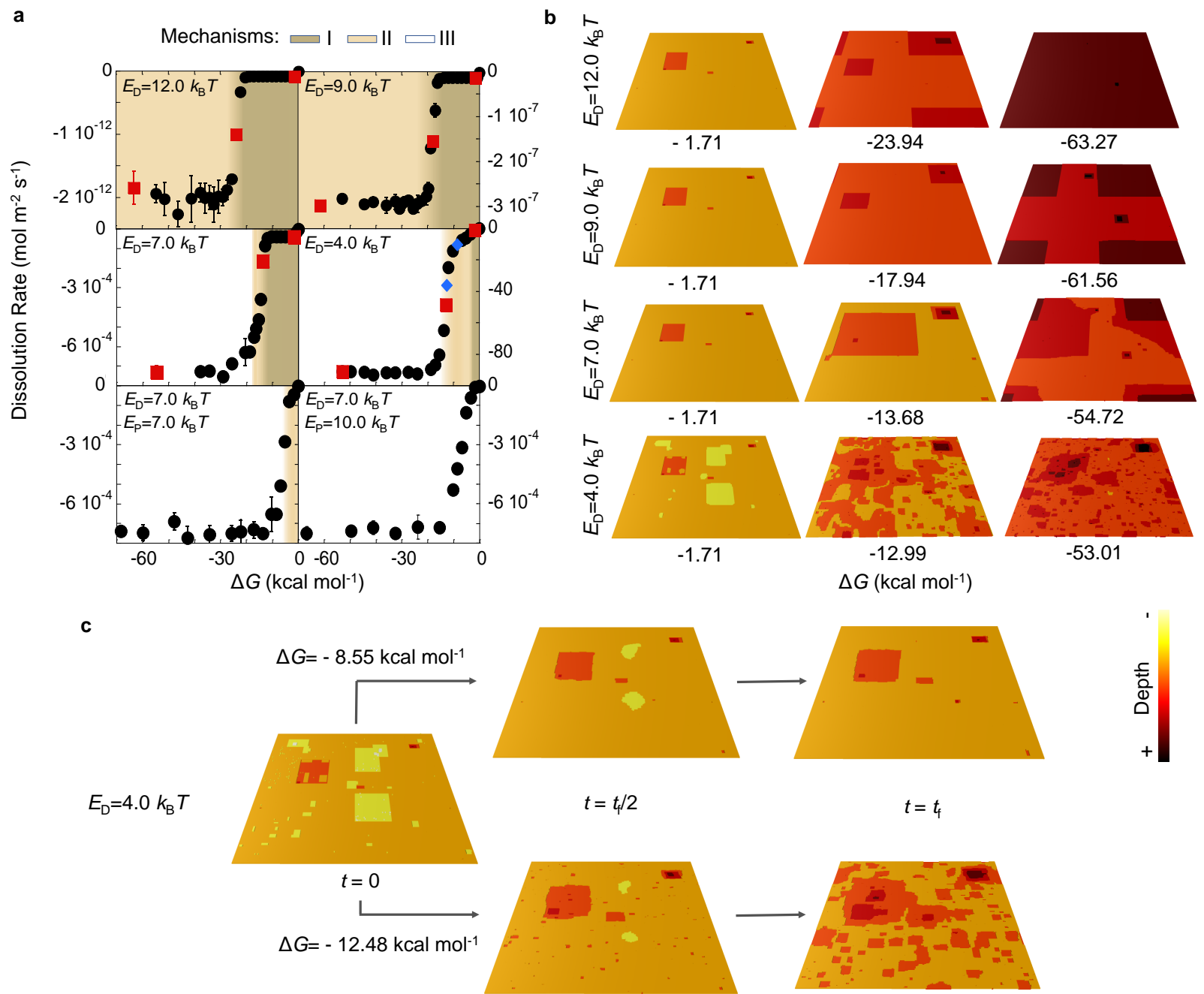

Figure 2: Dissolution rate versus Gibbs free energy $(\Delta G)$. a, Kossel crystal with different precipitation and dissolution energies. Points in red and blue are represented in panel $\mathrm{b}$ and $\mathrm{c}$ respectively. $\mathbf{b}$, Surfaces at a given time $t_{\mathrm{f}}$. $\mathbf{c}$, Time evolution of the surface. The visual representation is done using OVITO program. ${ }^{[42]}$. 
other hand, if $E_{\mathrm{P}}$ is significantly higher than $E_{\mathrm{D}}$, spontaneous vacancies can be created and pit opening is more favourable than adatom removal, provoking very irregular patterns. To our knowledge, this dissolution mode has not been proposed before and should deserve experimental validation. Though at present we cannot prove it, it seems plausible that this dissolution mode might be involved in the X-ray driven dissolution instabilities observed by Laanait et al. ${ }^{[43]}$ in Calcite-water interfaces.

In Figure $2 \mathrm{~b}$ dissolution patterns are represented in the three differentiated zones for the four cases in Figure 2a. Firstly, at high cohesive energies, $E_{\mathrm{D}}=12.0 k_{\mathrm{B}} T$ and $E_{\mathrm{D}}=9.0 k_{\mathrm{B}} T$, two dissolution mechanisms are easily differentiated. Close to equilibrium only terraces and adatoms are dissolved (mechanism I). Once $\Delta G_{\text {crit }}$ is reached, dissolution is produced almost exclusively from step retreat (mechanism II). Mechanism III does not exist in these cases. Secondly, looking at minerals with low cohesive energies $E_{\mathrm{D}}=7.0 k_{\mathrm{B}} T$ and $E_{\mathrm{D}}=4.0 k_{\mathrm{B}} T$, it can be seen that, like in the previous case, at close to equilibrium conditions adatoms and terraces are the only ones which are removed. Once $\Delta G_{\text {crit }}$ is reached pits start to open. But shortly after $\Delta G_{\text {crit }}$ value, spontaneous opening of surface (mechanism III) happens, the more the farther from equilibrium. At far from equilibrium conditions differences in energy between more and less coordinated atoms are not high enough to prevent them to dissolve closed to randomness, so very irregular patterns are produced.

The difference between mechanisms becomes more evident the lower the $E_{\mathrm{D}}$ is. Therefore, for a better sighting, in Figure 2c the dissolution pattern at three different times in the onset between mechanism II and mechanism III for a $E_{\mathrm{D}}=4.0 k_{\mathrm{B}} T$ mineral is shown. In minerals with low cohesive energy, in addition to the contribution to dissolution rate of the dislocation opening, there is certain contribution from mechanism III.

\subsection{Calculation of $\Delta G_{\text {crit }}$ as a function of $E_{\mathbf{D}}$ and $E_{\mathbf{P}}$}

Dislocations are key structural features for the mineral dissolution. It is validated in this work that in all studied cases dislocations produce the onset in the dissolution rate at $\Delta G_{\text {crit }}{ }^{[9,27,44,45]}$. Once the system is able to remove an atom close to dislocations, that is, with 4 neighbours in a Kossel crystal, the dissolution probability of the atoms in that layer increases due to the appearance of 3-coordinated kink site atoms. It is possible to analytically relate the microscopic variables $E_{\mathrm{D}}$ and $E_{\mathrm{P}}$ used in this model with the macroscopic $\Delta G_{\text {crit }}$ by Equation 8 demonstrated in the supplementary information:

$$
\Delta G_{\text {crit }}\left(k_{\mathrm{B}} T \text { units }\right) \approx\left(\alpha-b-N_{\mathrm{nD}} \cdot\left(E_{\mathrm{D}}-E_{\mathrm{P}}\right)\right) \cdot N_{\mathrm{nK}}
$$

where $N_{\mathrm{nD}}$ is the coordination number for an atom close to a dislocation (see Figure 1a,d), $N_{\mathrm{nD}}=4$ for a Kossel crystal. $\alpha$ value is determined in the supplementary information to report $\Delta G_{\text {crit }}$ at its middle height value: $\alpha=-2.3 \pm 0.9 k_{\mathrm{B}} T$. The accuracy of Equation 8 is checked in Table 1 . There is an excellent match between the $\Delta G_{\text {crit }}$ obtained from simulations and the analytically derived when the transition between mechanism I 


\begin{tabular}{|c|c|c|c|}
\hline$E_{\mathrm{D}}$ & $E_{\mathrm{P}}$ & Simulation $\Delta G_{\text {crit }}\left(\mathrm{kcal} \mathrm{mol}^{-1}\right)$ & Analytical $\Delta G_{\text {crit }}\left(\mathrm{kcal} \mathrm{mol}^{-1}\right)$ \\
\hline 12.0 & 1.0 & -24 & -23.8 \\
9.0 & 1.0 & -18 & -18.4 \\
7.0 & 1.0 & -15 & -14.9 \\
7.0 & 7.0 & -2 & -4.1 \\
7.0 & 10.0 & -7 & 1.3 \\
4.0 & 1.0 & -13 & -9.5 \\
\hline
\end{tabular}

Table 1: Validation of Equation 8. $\Delta G_{\text {crit }}$ is obtained by applying the Equation 10, which is later explained, to Figure $2 \mathrm{~b} . E_{\mathrm{D}}$ and $E_{\mathrm{P}}$ are in $k_{\mathrm{B}} T$ units. Error in analytical $\Delta G_{\text {crit }}$ is $\pm 1.6 \mathrm{kcal} \mathrm{mol}^{-1}$ (see Figure $\mathrm{S} 2$ ).

and II is given at negative $\Delta G$. It must be noted that according to expression 8 , for a given mineral with a fix $E_{\mathrm{D}}, \Delta G_{\text {crit }}$ also depends on $E_{\mathrm{P}}$. Therefore different dissolution mechanisms and rates can be experimentally reported even with the same mineral and $\Delta G$. It is also interesting to point that the analytical expression allows to determine the theoretical $\Delta G_{\text {crit }}$ even in conditions where $E_{\mathrm{P}}$ is higher than $E_{\mathrm{D}}$ and the onset is produced at positive $\Delta G$. Indeed, this behaviour has been observed by Juilland and Galluci in alite ${ }^{[10]}$.

\subsection{Relationship between $E_{\mathrm{D}}$ and $E_{\mathrm{a}}$}

TST proposes dissolution as a set of elementary reactions that can take place if the system is able to overcome an energy barrier determined by an Arrhenius equation ${ }^{[1]}$.

$$
R=A^{\prime} \cdot \exp \left(-\frac{E_{\mathrm{a}}}{k_{\mathrm{B}} \cdot T}\right)
$$

Where $A^{\prime}$ is a constant.

The activation energy in the Arrhenius equation is in fact an apparent energy because it compiles the contribution from many terms and not just the energy barrier of the elementary reactions. In this sense, the supplementary information includes a deeper study concerning the fundamental frequency dependence with temperature, which is closely related to the dissolution rate (see Equation 1 ).

In the idealized simulation model the activation energy $E_{\mathrm{a}}$ can be written as $E_{\mathrm{a}}=$ $N_{\mathrm{nK}} \cdot E_{\mathrm{D}}$ because it is observed experimentally that the sublimation energy of a crystal corresponds to the change of internal energy when detaching a kink atom. Nevertheless, as it is checked below, this relation is not true at the whole $\Delta G$ range $^{[22]}$. In the next step, the real link between macroscopic $E_{\mathrm{a}}$ and microscopic $E_{\mathrm{D}}$ is explored to compare with experimental data.

Experimental studies usually report a single $E_{\mathrm{a}}$, usually corresponding to far from equilibrium conditions in a grain shape system. Nevertheless, in an infinite surface system $E_{\mathrm{a}}$ actually depends on the dissolution mechanism. In the mechanism I zone, dissolution 

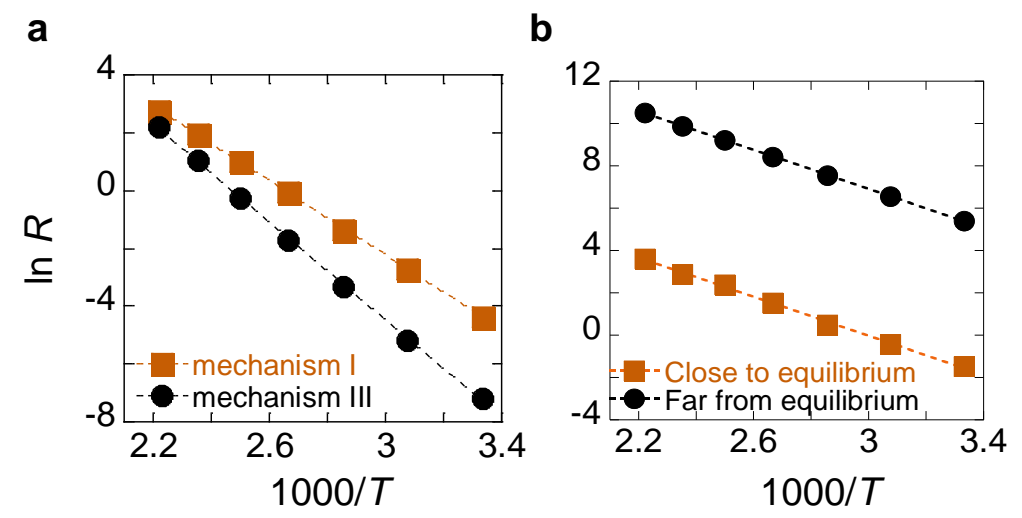

Figure 3: Natural logarithm of dissolution rate $\ln R$ versus 1000/T. a, Kossel crystal of $E_{\mathrm{D}}=7.0 k_{\mathrm{B}} T$ and $E_{\mathrm{P}}=1.0 k_{\mathrm{B}} T$ units. $\mathbf{b}$, Same values for a diamond like mineral. Points in black represent far from equilibrium conditions. Points in orange represent close to equilibrium conditions. Error is smaller than the size of the points. Arrhenius fitting parameters are represented in Table 2.

\begin{tabular}{|l|c|c|c|}
\cline { 3 - 4 } \multicolumn{1}{c|}{} & Kossel crystal & Diamond like system \\
\hline \multirow{3}{*}{ mech. III / f.f.e } & $\ln A^{\prime}$ & $20.98 \pm 0.19$ & $20.69 \pm 0.05$ \\
& $\frac{E_{\mathrm{a}}}{E_{\mathrm{D}}}$ & 4.0 & 2.2 \\
& $E_{\mathrm{a}}$ & $70.5 \pm 0.6 \mathrm{~kJ} \mathrm{~mol}^{-1}$ & $38.0 \pm 0.2 \mathrm{~kJ} \mathrm{~mol}^{-1}$ \\
\hline \multirow{3}{*}{ mech. I / c.e } & $\ln A^{\prime}$ & $17.15 \pm 0.06$ & $13.8 \pm 0.3$ \\
& $\frac{E_{\mathrm{a}}}{E_{\mathrm{D}}}$ & 3.1 & 2.2 \\
& $E_{\mathrm{a}}$ & $53.73 \pm 0.17 \mathrm{~kJ} \mathrm{~mol}^{-1}$ & $38.3 \pm 0.9 \mathrm{~kJ} \mathrm{~mol}^{-1}$ \\
\hline
\end{tabular}

Table 2: Activation energy and fitting parameters. Reported values in Figure 3 for both, a Kossel crystal in mechanisms III and I, and a four coordinated diamond like mineral at far from equilibrium (f.f.e) and close to equilibrium conditions (c.e). The latter is studied in the supplementary information. 
is driven by removal of kink atoms in terraces. In contrast, in mechanisms II/III zones dissolution is driven by step retreat. Therefore, microscopically it is expected that $E_{\mathrm{a} \text { m.I }} \approx$ $N_{\mathrm{nK}} \cdot E_{\mathrm{D}}$ and $E_{\mathrm{a} \text { m.II/III }} \approx N_{\mathrm{nD}} \cdot E_{\mathrm{D}}$, where $N_{\mathrm{nK}}$ and $N_{\mathrm{nD}}$ are 3 and 4 respectively for a Kossel crystal (see Figure 3a and Table 2).

In order to verify such relationship, Arrhenius equation has been used to fit the natural logarithm of the dissolution rate $\ln R$ as a function of the inverse of $T$ for fixed $\Delta G$ conditions corresponding to mechanisms I and III. For that, values of $7 k_{\mathrm{B}} T$ and $1 k_{\mathrm{B}} T$ units have been chosen for $E_{\mathrm{D}}$ and $E_{\mathrm{P}}$ respectively. For the mechanism III, the Kossel crystal described before has been used, and the dissolution rate at each $T$ is taken as the plateau value at far from equilibrium conditions. However, to study mechanism I, a limiting value of the reaction rate $R$ is not reached. for this case, the beginning of the $\Delta G_{\text {crit }}$ onset represents a good reference point since no contributions from other mechanisms are involved. In addition, the Kossel crystal topography has been modify for a better quantification of the rate. As only terraces dissolution is the governing mechanism, a surface with a big terrace of 8 sites high has been constructed without any other surface defect.

As it can be seen in Figure 3a, there is good linear correlation of dissolution rate logarithm with the inverse of temperature for both mechanisms, which indicates that the systems are following an Arrhenius process as expected. An important consequence is that the expected values of $E_{a}$ as a function of $E_{D}$ are obtained $\left(E_{\mathrm{a} \text { m.I }}=3.1 \cdot E_{\mathrm{D}}\right.$ and $\left.E_{\text {a m.II/III }}=4.0 \cdot E_{\mathrm{D}}\right)$. Therefore, the macroscopic dissolution activation energy can be obtained directly from the computed dissolution reaction energy in any $\Delta G$ conditions.

\subsection{Comparison with experimental dissolution rates}

Next target is to apply the proposed model to describe the experimentally reported dissolution for several minerals: albite ${ }^{[12,27]}$, smectite ${ }^{[13,27]}$, alite ${ }^{[17]}$, labradorite ${ }^{[18,27]}$ and Kfeldspar ${ }^{[46]}$. Table 3 contains the obtained values for the model parameters in Equations 2 and 3 , their activation energies, and the $\Delta G_{\text {crit }}$.

The initial values for the simulations have been set as follows. First the $\Delta G_{\text {crit }}$ value is obtained from the empirical Equation 10. Note that both this empirical equation and Equation 8 report $\Delta G_{\text {crit }}$ at the middle height value. Second, a guess $E_{\mathrm{D}}$ value is chosen based on the dissolution rate limit value at far from equilibrium conditions. Finally, $E_{\mathrm{P}}$ is calculated from Equation 8. With these initial values simulations are run and the parameters are adjusted $a d-h o c$ to obtain the best possible fit according to the regression coefficient between the simulation results and an empirical fit for the experimental data.

The experimental and computed dissolution rates as a function of $\Delta G$ are shown in Figure 4. Besides, an empirical fitting function that differs from the previously proposed in the state of the art ${ }^{[12,34]}$ is shown. The fitting function adds a logistic function term ${ }^{[47]}$ to the TST expression, and reads as in Equation 10: 


$$
\begin{aligned}
R= & A \cdot\left(1-\exp \left(\frac{\Delta G}{k_{\mathrm{B}} \cdot T}\right)\right)+ \\
& B \cdot\left(1-\frac{1}{1+\exp \left(D \cdot\left(-\Delta G+\Delta G_{\text {crit }}\right)\right)}\right)
\end{aligned}
$$

where $A$ and $B$ are the limiting dissolution rates corresponding to close and far from equilibrium conditions respectively, and $D$ is a fitting parameter related to $E_{\mathrm{D}}$ and topography.

From Figure 4 it can be first noticed that the sigmoidal shape of the mineral dissolution rate as a function of $\Delta G$ can be reproduced with the presented model. Furthermore, Equation 8 provides a $\Delta G_{\text {crit }}$ in good agreement with the experimental and computed values and helps to define the onset in cases in which transition between mechanism I and II is not so clear, such as the case of K-feldspar.

The dissolution and precipitation energies $E_{D}$ and $E_{P}$, the activation energies in mechanism I and II regions, and the $\Delta G_{\text {crit }}$ obtained from the fitting are presented in Table 3 , together with experimental dissolution activation energies. All the obtained values are in reasonable orders of magnitude, yet the experimental dissolution activation energies are consistently overestimated by nearly a factor 2 for all minerals. Clearly, using a Kossel crystal with the same topography for all the studied minerals will necessary lead to qualitative results. In addition, there is a dependency of the dissolution rate with the dislocation density ${ }^{[37,48-51]}$. Such dependency has been tested with the presented model and reveals that the dislocation density is in particular important for high $E_{\mathrm{D}}$ minerals at far from equilibrium conditions (see supplementary information). Other discrepancies in the values can arise from considering a $\Delta G$ value independent of ion activities, the unknown initial surface conditions, or the homogeneous fundamental frequency value chosen for the study.

\section{Conclusions}

In this work a new atomistic mineral dissolution model is presented. Based on a KMC protocol, the main novelty of the model consists in taking into account the microscopic reversibility of chemical reaction by adding a new precipitation term to the usual TST dissolution equation. As a result, it is possible for the first time to reproduce the experimentally observed sigmoidal dependence of the dissolution rate over the whole $\Delta G$ range by atomistic simulations. Indeed, as proof of concept, the two main parameters of the model (the dissolution and precipitation energies) have been calibrated to correctly fit the dissolution rates of several representative minerals. Besides, and what is most important, the model successfully captures the proposed dissolution mechanisms and their activation. The three observed dissolution mechanisms naturally emerge from the simulations 


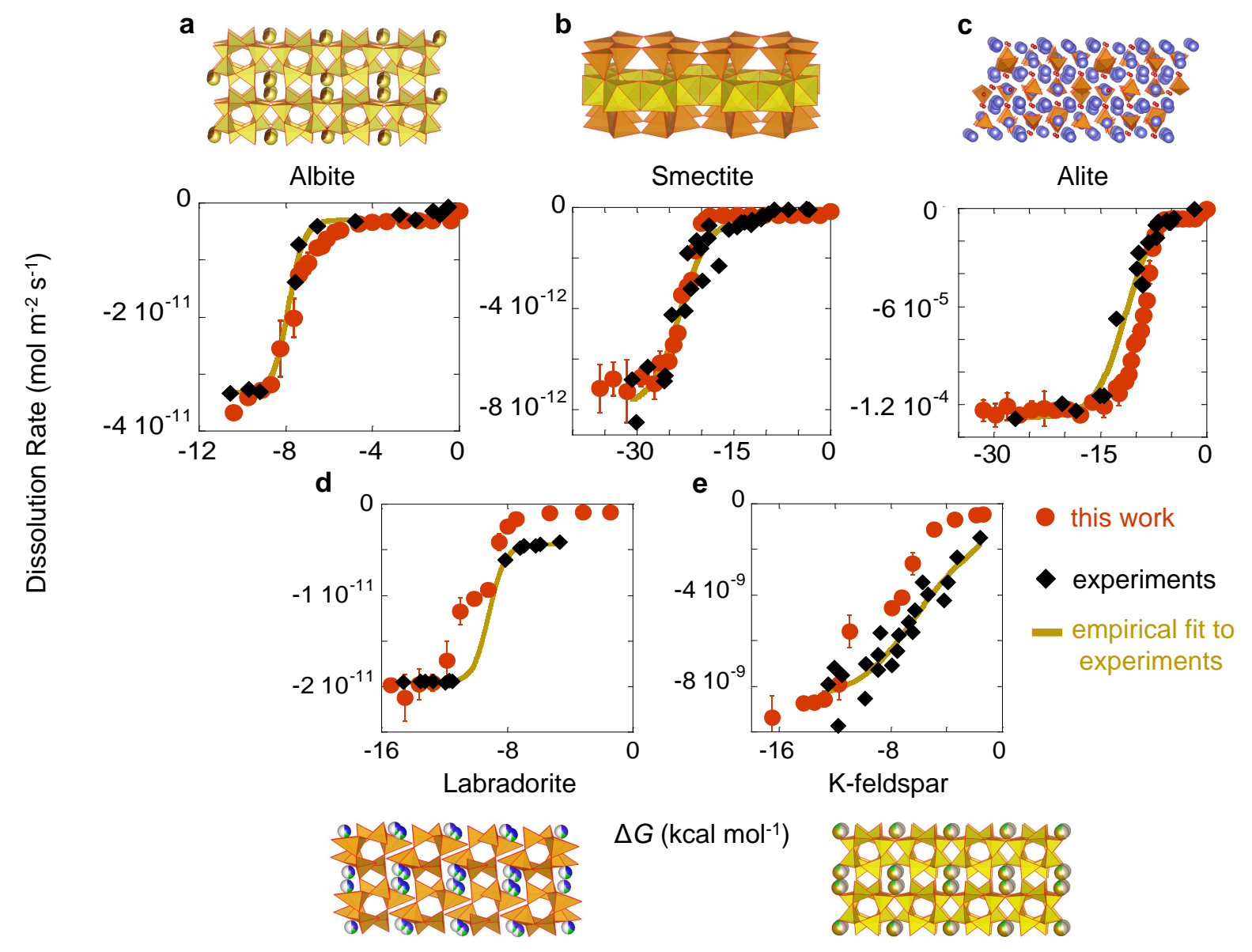

Figure 4: Comparison of the experimental data with the proposed dissolution theory. a, Albite at $\mathrm{pH} 8.8$ and $357 \mathrm{~K}^{[12,27]}$. b, Smectite at $\mathrm{pH} 3$ and $357 \mathrm{~K}^{[13,27]}$. c, Alite at $300 \mathrm{~K}^{[17]}$. d, Labradorite at $\mathrm{pH} 3$ and $300 \mathrm{~K}^{[18,27]}$. e, K-feldspar at $\mathrm{pH} 9$ and 423 $\mathrm{K}^{[46]}$. Structural visualization is done using VESTA program ${ }^{[52]}$. 


\begin{tabular}{|l|c|c|c|c|c|c|c|c|}
\hline Mineral & $T(\mathrm{~K})$ & $E_{\mathrm{D}}\left(k_{\mathrm{B}} T\right)$ & $E_{\mathrm{P}}\left(k_{\mathrm{B}} T\right)$ & $\begin{array}{c}E_{\mathrm{a} \text { m.II/III }} \\
(\mathrm{kJ} \\
\left.\mathrm{mol}^{-1}\right)\end{array}$ & $\begin{array}{c}E_{\mathrm{a} \mathrm{m} . \mathrm{I}} \\
(\mathrm{kJ} \\
\left.\mathrm{mol}^{-1}\right)\end{array}$ & $\begin{array}{c}E_{\mathrm{a} \text { exp. }} \\
(\mathrm{kJ} \\
\left.\mathrm{mol}^{-1}\right)\end{array}$ & $\begin{array}{c}\text { Simulation } \\
\Delta G_{\text {crit }} \\
\left(\mathrm{kcal} \mathrm{mol}^{-1}\right)\end{array}$ & $\begin{array}{c}\text { Analytical } \\
\Delta G_{\text {crit }} \\
\left(\mathrm{kcal} \mathrm{mol}^{-1}\right)\end{array}$ \\
\hline Albite & 357 & 11.14 & 10.00 & 132 & 99 & $\begin{array}{c}71 \pm 7^{[54]} \\
88.6^{[55]}\end{array}$ & -8 & $-7.3 \pm 1.9$ \\
Smectite & 357 & 11.73 & 4.00 & 139 & 104 & $52 \pm 4^{[57]}$ & -23 & $-21.4 \pm 1.9$ \\
Alite & 300 & 7.47 & 4.95 & 75 & 56 & $49^{*}[10]$ & -11 & $-8.6 \pm 1.6$ \\
Labradorite & 300 & 11.40 & 8.8 & 114 & 85 & $45.2^{[53]}$ & -9 & $-8.8 \pm 1.6$ \\
Feldspar & 423 & 9.95 & 10.3 & 139 & 104 & $38.0^{[53]}$ & -6 & $-5 \pm 2$ \\
\hline
\end{tabular}

Table 3: Model parameters for minerals. Fitting values for Figure 4 of the corresponding activation energy in each mechanism and comparison with bibliographic ones. $\Delta G_{\text {crit }}$ value obtained by Equation 10 and analytically by Equation 8. (*) This value is obtained experimentally in a plane with dislocation opening.

depending on dissolving energy $E_{\mathrm{D}}$ and $\Delta G$ : initial irregularities dissolution (mechanism I) at close to equilibrium conditions, pit opening and step retreat (mechanism II) when $\Delta G_{\text {crit }}$ is reached, and spontaneous vacancy opening (mechanism III) at far from equilibrium conditions when the $E_{\mathrm{D}}$ is low enough. The model also confirms the generally accepted idea that the onset for the dissolution rate increase is originated by the opening of pits, which constantly supplies terraces for step retreat. Interestingly, according to the simulations, when the dissolution and precipitation energies are sufficiently low and high respectively, there can exist close-to-equilibrium dissolution modes where spontaneous vacancies creation and pit opening can occur before adatom and terrace removal. These dissolution modes have not been previously reported, and should deserve due experimental attention.

In summary, the present model sheds new light on the subtle dissolution mechanisms, and can open the door to the development of a comprehensive theoretical framework for dissolution and other surface-related phenomena like etching ${ }^{[58]}$. Future works will be focused on building realistic mineral models beyond the current implementation based on simple Kossel crystals.

\section{References}

[1] N. Van Breemen, P. Buurman, Soil formation, Springer Science \& Business Media, 2002.

[2] K. Kalbitz, S. Solinger, J.-H. Park, B. Michalzik, E. Matzner, Soil science 2000, 165, 277-304.

[3] H. R. Krouse, C. A. Viau, L. S. Eliuk, A. Ueda, S. Halas, Nature 1988, 333, 415. 
[4] M. Canals, J. D. Meunier, Geochimica et Cosmochimica Acta 1995, 59, 699-709.

[5] R. Lal, Philosophical Transactions of the Royal Society of London B: Biological Sciences 2008, 363, 815-830.

[6] R. Lal, Science 2004, 304, 1623-1627.

[7] S. Blain, B. Quéguiner, L. Armand, S. Belviso, B. Bombled, L. Bopp, A. Bowie, C. Brunet, C. Brussaard, F. Carlotti, et al., Nature 2007, 446, 1070.

[8] D. E. Giammar, R. G. Bruant Jr, C. A. Peters, Chemical Geology 2005, 217, 257276.

[9] P. Juilland, E. Gallucci, R. Flatt, K. Scrivener, Cement and Concrete Research 2010, $40,831-844$.

[10] P. Juilland, E. Gallucci, Cement and Concrete Research 2015, 76, 180-191.

[11] A. C. Lasaga, Kinetic theory in the earth sciences, Princeton university press, 2014.

[12] T. Burch, K. Nagy, A. Lasaga, Chemical Geology 1993, 105, 137 - 162.

[13] J. Cama, J. Ganor, C. Ayora, C. A. Lasaga, Geochimica et Cosmochimica Acta 2000, 64, 2701-2717.

[14] J. Xu, C. Fan, H. H. Teng, Chemical geology 2012, 322, 11-18.

[15] R. S. Arvidson, A. Luttge, Chemical Geology 2010, 269, 79-88.

[16] L. Nicoleau, M. A. Bertolim, Journal of the American Ceramic Society 2016, 99, $773-786$.

[17] L. Nicoleau, A. Nonat, D. Perrey, Cement and Concrete Research 2013, 47, 14-30.

[18] A. S. Taylor, J. D. Blum, A. C. Lasaga, Geochimica et Cosmochimica Acta 2000, 64, 2389-2400.

[19] A. Lüttge, Journal of Electron Spectroscopy and Related Phenomena 2006, 150, 248259.

[20] C. Fischer, I. Kurganskaya, A. Luttge, Applied geochemistry 2018, 91, 140-148.

[21] A. C. Lasaga, A. E. Blum, Geochimica et Cosmochimica Acta 1986, 50, 2363-2379.

[22] A. C. Lasaga, A. Luttge, American Mineralogist 2004, 89, 527-540.

[23] A. F. Voter in Radiation effects in solids, Springer, 2007, pp. 1-23. 
[24] Y. Liang, D. R. Baer, J. M. McCoy, J. E. Amonette, J. P. Lafemina, Geochimica et Cosmochimica Acta 1996, 60, 4883-4887.

[25] I. Kurganskaya, A. Luttge, The Journal of Physical Chemistry C 2013, 117, 2489424906.

[26] I. Kurganskaya, A. Luttge, The Journal of Physical Chemistry C 2016, 120, 64826492.

[27] A. C. Lasaga, A. Luttge, Science 2001, 291, 2400-2404.

[28] P. Meakin, K. M. Rosso, Journal of Chemical Physics 2008, 129.

[29] L. Briese, R. S. Arvidson, A. Luttge, Geochimica et Cosmochimica Acta 2017, 212, $167-175$.

[30] C. Fischer, A. Luttge, Proceedings of the National Academy of Sciences 2018, 115, 897-902.

[31] L. Zhang, A. Luttge, American Mineralogist 2007, 92, 1316-1324.

[32] L. Zhang, A. Lüttge, Geochimica et Cosmochimica Acta 2009, 73, 2832-2849.

[33] K. Oura, M. Katayama, A. Saranin, V. Lifshits, A. Zotov, Surface Science, Vol. 602, 2003.

[34] S. L. Brantley, J. D. Kubicki, A. F. White, Kinetics of water-rock interaction, Vol. 168, Springer, 2008.

[35] J. Z. Bandstra, S. L. Brantley, Geochimica et Cosmochimica Acta 2008, 72, 25872600 .

[36] G. V. Gibbs, R. T. Downs, D. F. Cox, N. L. Ross, C. T. Prewitt, K. M. Rosso, T. Lippmann, A. Kirfel, Zeitschrift für Kristallographie-Crystalline Materials 2008, 223, 01-40.

[37] H. Teng, Geochimica et Cosmochimica Acta 2004, 68, 253-262.

[38] A. Chatterjee, A. F. Voter, The Journal of chemical physics 2010, 132, 194101.

[39] E. C. Dybeck, C. P. Plaisance, M. Neurock, Journal of chemical theory and computation 2017, 13, 1525-1538.

[40] M. Jørgensen, H. Grönbeck, The Journal of chemical physics 2018, 149, 114101.

[41] A. Putnis, An introduction to mineral sciences, Cambridge University Press, 1992. 
[42] A. Stukowski, Modelling and Simulation in Materials Science and Engineering 2010, $18,015012$.

[43] N. Laanait, E. B. Callagon, Z. Zhang, N. C. Sturchio, S. S. Lee, P. Fenter, Science 2015, 349, 1330-1334.

[44] P. M. Dove, N. Han, J. J. De Yoreo, Proceedings of the National Academy of Sciences 2005, 102, 15357-15362.

[45] C. Fischer, R. S. Arvidson, A. Lüttge, Geochimica et Cosmochimica Acta 2012, 98, $177-185$.

[46] J.-M. Gautier, E. H. Oelkers, J. Schott, Geochimica et Cosmochimica Acta 1994, 58, 4549-4560.

[47] D. G. Zill, A first course in differential equations with modeling applications, Cengage Learning, 2012.

[48] G. Holdren, W. Casey, H. Westrich, M. Carr, M. Boslough, Chemical Geology 1988, 70,79 .

[49] W. H. Casey, M. J. Carr, R. A. Graham, Geochimica et Cosmochimica Acta 1988, 52, 1545-1556.

[50] J. Schott, S. Brantley, D. Crerar, C. Guy, M. Borcsik, C. Willaime, Geochimica et Cosmochimica Acta 1989, 53, 373-382.

[51] R. T. Cygan, W. H. Casey, M. B. Boslough, H. R. Westrich, M. J. Carr, G. R. Holdren Jr, Chemical Geology 1989, 78, 229-244.

[52] K. Momma, F. Izumi, Journal of Applied Crystallography 2008, 41, 653-658.

[53] J. L. Palandri, Y. K. Kharaka, A compilation of rate parameters of water-mineral interaction kinetics for application to geochemical modeling, Tech. Rep., Geological Survey Menlo Park CA, 2004.

[54] N. Rose, Geochimica et Cosmochimica Acta 1991, 55, 3273-3286.

[55] H. C. Helgeson, W. M. Murphy, P. Aagaard, Geochimica et Cosmochimica Acta 1984, 48, 2405-2432.

[56] Y. Chen, S. L. Brantley, Chemical Geology 1997, 135, 275-290.

[57] A. Bauer, G. Berger, Applied Geochemistry 1998, 13, 905-916.

[58] X. Ye, M. R. Jones, L. B. Frechette, Q. Chen, A. S. Powers, P. Ercius, G. Dunn, G. M. Rotskoff, S. C. Nguyen, V. P. Adiga, et al., Science 2016, 354, 874-877. 


\section{Acknowledgments}

This work has been done under the umbrella of the Basque Country Initiative for Cement and Concrete Research (BASKRETE), and supported by the ELKARTEK and EMAITEK programs of the Basque Country Government. JSD also acknowledges the funding from the Spanish Ministry of Economy, Industry and Competitiveness (project Ref-201860I057) and the Spanish Ministry of Science, Innovation and Universities (project Ref RTI2018-098554-B-I00). P. Martin acknowledges support from the PhD scholarship Tecnalia Research \& Innovation's grant. All the simulations have been carried out at the high performance computing service of Basque Country i2basque.

\section{Keywords}

Mineral dissolution; Kinetic Montecarlo; Gibbs free energy; Transition State Theory.

\section{Competing Interests}

The authors declare that they have no competing financial interests. 